\title{
Multiframe Ultrasonic TOFD Weld Inspection Imaging Based on Wavelet Transform and Image Registration
}

\author{
Jiaxu Duan $\mathbb{D}^{1,2}$ Lin Luo $\mathbb{D}^{1,2}$ Xiaorong Gao, ${ }^{1,2}$ Jianping Peng, ${ }^{1,2}$ and Jinlong $\mathrm{Li}^{1,2}$ \\ ${ }^{1}$ Photoelectric Engineering Institute, Southwest Jiaotong University, Chengdu 610031, China \\ ${ }^{2}$ Southwest Jiaotong University NDT Research Center \& Olympus NDT Joint Laboratory of Nondestructive Testing, \\ Chengdu 610031, China
}

Correspondence should be addressed to Lin Luo; happyluolin@vip.163.com

Received 30 May 2017; Revised 14 November 2017; Accepted 3 December 2017; Published 13 February 2018

Academic Editor: Jaime Lloret

Copyright (c) 2018 Jiaxu Duan et al. This is an open access article distributed under the Creative Commons Attribution License, which permits unrestricted use, distribution, and reproduction in any medium, provided the original work is properly cited.

TOFD (time of flight diffraction) is a kind of weld defect detection technology by using ultrasonic diffraction wave signal. Because the diffraction intensity is far less than ultrasonic echo wave intensity, thus, the noise contained in TOFD signal is fairly large, and the formed image is not clear enough. Therefore, it is difficult to determine the size of defects accurately. In this paper, a method of noise reduction of TOFD signal and improving the resolution of the image are discussed based on the combination of wavelet thresholding and image registration. Wavelet multiresolution analysis method is adopted and the A-scan signal is decomposed into different frequency components. We propose a new threshold function to process the wavelet coefficients, which guarantees to denoise while preserving the useful information as much as possible. Setting up the ultrasonic TOFD inspection system and the image data with randomly distributed noise can be obtained via fine shake of the probes during testing. Then, image registration based on maximum correlation and blending is adopted to eliminate the noise in further step. The result shows that the proposed method can achieve denoising, together with resolution enhancement.

\section{Introduction}

Ultrasonic time of flight diffraction defect inspection was introduced by Silk [1] in 1970s, and this technique is being widely applied in weld defect inspection in NDT field from then on. TOFD inspection has the property of high accuracy, high reliability, and especially the adequate location of the defect [2]. Ultrasonic TOFD technique uses the diffraction echo to determine the defects quantitatively. In general, the diffraction echo signal is weak and is easily affected by the noise. In order to effectively detect weak signal obtained from the diffraction signal, TOFD detection system is always operated in high-gain mode, which increase not only the signal intensity but also the noise. As a result, the TOFD images always contain abundant noise and low signal-to-noise ratio (SNR), which brings significantly negative effect on defect detection. In order to overcome this shortcoming, researchers have done a lot of optimizations, for instance, using image-processing techniques based on crosscorrelation to improve the accuracy of measuring the defect size
[3], adjusting distance between probes and the angle of the probes to obtain the defect location more accurately [4], using support vector machine classifier to determine the pattern of the defects [5], using a local optimal threshold technique to extract the crack edges in TOFD image [6], flaw detection in TOFD images based on texture features [7], automating crack detection by analyzing the curve formed by the sparse matrix elements [8], and using the finite element technique to simulate the ultrasonic TOFD data [9]. In terms of signal processing, the following methods can be used: embedded signal identification technique (ESIT) [10], the wavelet packet method [11], the Hilbert transform method [12], and so on. Wavelet multiresolution analysis is an effective method to reduce the noise in images. Due to the high correlation between the diffracted wave and its wavelet coefficients, the wavelet denoising can achieve good robustness [13]. The denoise result via wavelet transform is highly dependent on the order of the wavelet. With the increasing of the order of the wavelet, better performance can be obtained, while the heavier computation burden is 
brought simultaneously. Image decomposition can be done by using $2 \mathrm{D}$ wavelet transform to obtain the wavelet coefficients; then, the threshold function is adopted to compress the wavelet coefficients, and the denoised wavelet coefficients can be achieved. Finally, the denoised image is obtained after image reconstruction via inverse wavelet transform [14]. Hoseini et al. [11] adopts stationary wavelet transform to decompose the ultrasonic image into the components with different frequencies and directions; then, the wavelet coefficients in horizontal, perpendicular, and diagonal are calculated. According to [15], taking the efficiency into consideration, only the detail components of wavelet coefficients are considered due to the noise majorly exists in this part and retains the approximate components. After the wavelet transform procedure, the noise can be eliminated effectively. In [16], soft thresholding is adopted as the basic method and an advanced algorithm is proposed by modifying the basic method.

This paper discusses a concept of multiframe model and adopts multiple TOFD images, in the sense of the combination of wavelet transform and image registration, SNR and the quality of the image can be enhanced by blending the denoised images together. The remaining of this paper is arranged as follows: in Section 2, we discuss and analyze the principle of imaging procedure of ultrasonic TOFD inspection, wavelet transform, and image registration. Section 3 proposes and analyzes a new threshold function. The experiment system is introduced in the experimental part, and the experiment result is analyzed and evaluated by using SNR, crosscorrelation, and mean square error (MSE) as well.

\section{Principle}

2.1. TOFD Imaging. Ultrasonic TOFD inspection is a kind of defect-testing technique using ultrasonic diffraction signal. Figure 1 shows the schematic diagram of the principle of TOFD detection, which is operated under the theory of calculating the time difference of the echo wave propagated between the two ends of the crack. Diffracted wave and the echo wave are physical phenomenon. The difference between them is that the former is generated during the propagation on the border of two different media. Once there are some defects during the ultrasound propagating, thereafter, diffracted wave will be generated on the ends of the crack. The more obvious the diffraction phenomenon is, the sharper the crack is. Actually, the echo wave is much stronger than the diffracted wave; moreover, the diffracted wave is propagated randomly, which means there is not a certain direction for wave propagation $L \mathrm{n}$.

The inspection system shown in Figure 1 contains a pair of longitudinal wave probe with the same frequency, wafer size, and diffraction angle. The distance between the two probes is determined by the thickness of the specimen, the testing frequency, and the beam angle. One probe of the probe pair is used as the transmitter, while the other is the receiver. The $\mathrm{D}$-scan image data is generated by moving the probe pair during inspection. If there is no defect in the specimen, the receiver will receive two echo waves with inverse phase, one of them is the lateral wave propagated along the

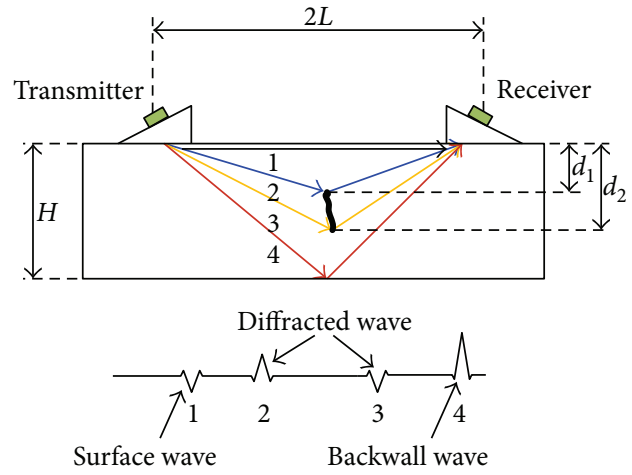

FIGURE 1: Ultrasonic TOFD inspection schematic diagram.

surface of the specimen and the other is the echo wave from the bottom. If the specimen contains one or more defects, the diffracted wave will be formed among the above two waves, and the phase difference between the two ends on the crack is $180^{\circ}$. The depth between the upper end $\left(d_{1}\right.$ in Figure 1$)$ and the lower end $\left(d_{2}\right.$ in Figure 1$)$ can be calculated as follows:

$$
d=\frac{\sqrt{(v \Delta t)^{2}+4 v L \Delta t}}{2},
$$

where $v$ represents the speed of the longitudinal wave; $\Delta t$ denotes the time difference between diffracted wave; $L$ denotes the half distance between two probes.

In the testing procedure of TOFD system, in order to solve the problem of difficult recognition of echo wave, longitudinal wave can be adopted but not lateral wave. The calculation result of the depth of the defect is unique via longitudinal wave and its speed in different modes of the wave have different speeds in the same specimen, and the fastest one is the longitudinal wave. In the propagation of the wave in steel, the speed of the longitudinal wave is twice as much as the lateral wave. Consequently, the earliest received signal is the longitudinal wave. Ultrasonic TOFD inspection can be proceeded by A-scan, and the B- or Dscan signal can be synthesized by A-scan signal. B-scan and $\mathrm{D}$-scan signal is $2 \mathrm{D}$ image and is generated if the probe pair is moved parallel and perpendicular to the middle line of the weld, respectively. The weakness of the diffracted wave and the inhomogeneity of specimen structure makes the observed signal always contains lots of noise. For the convenience of observation of the diffracted wave signal, it is necessary for the system to be operated under the high-gain mode. As a result, the useful signal and the noise are enhanced together; thus, the formed TOFD image is always with low SNR.

2.2. Wavelet Transform. According to the theory of wavelet analysis proposed by Mallat [17], the relationship between coefficients can be presented by multisampling filter; thus, it is easy to adopt digital signal processing analysis. Multisampling filter can decompose the signal into multiple components with different frequencies. The observed signal can be presented as follows: 


$$
\begin{aligned}
f(x)= & \frac{1}{\sqrt{M}} \cdot \sum_{k} c_{j 0, k}(x) \Phi_{j 0, k}(x) \\
& +\frac{1}{\sqrt{M}} \cdot \sum_{j \leq j 0} \sum_{k} d_{j, k}(x) \Psi_{j, k}(x), \\
\Phi_{m, k}(x)= & \sum_{n} h_{n} \sqrt{2} \cdot \Phi_{m+1,2 k+n}(2 x-n), \\
\Psi_{m, k}(x)= & \sum_{n} g_{n} \sqrt{2} \cdot \Phi_{m+1,2 k+n}(2 x-n),
\end{aligned}
$$

where $\Phi_{m, k}(x)$ and $\Psi_{m, k}(x)$ denote the scale function and the wavelet function, respectively; $c_{j 0, k}$ and $d_{j, k}$ denote two filters which satisfy the condition of scale difference function. $m$ and $n$ denote the number of sampling points, while $k$ denotes the order of decomposition. $h_{n}=\left\langle\Psi_{0,0}, \Phi_{-1, n}\right\rangle$ and $g_{n}=\left\langle\Phi_{0,0}, \Phi_{-1, n}\right\rangle$ represent the wavelet coefficients with higher frequencies and lower frequencies.

In order to achieve the restored signal with much less noise than before, we combine the high-frequency components after processing and the retained low-frequency components together. Thresholding is one of the most widely used methods to compress the noise in high-frequency components, including hard thresholding and soft thresholding. In the procedure of hard thresholding, the discontinuity at critical points can lead to bell effect; moreover, the pixel value ranges between two critical points are all substituted to 0 , which makes the image information lost severely and results to the loss of image fidelity. Soft thresholding is more flexible compared to hard thresholding. However, the soft thresholding could not give an optimum value for the calibration of the higher coefficients, and the smoothness of the soft thresholding always reduces the resolution and the definition of images.

In order to overcome the shortcomings in classic threshold functions, the variance of the noise signal should be estimated based on Bayesian statistics before adopting thresholding on the wavelet coefficients. Assuming that the wavelet coefficient in high-frequency components yields to Gaussian distribution, thus the posterior probability can be presented as follows:

$$
P\left(h_{n, i} \mid \theta\right)=\frac{1}{\sqrt{2 \pi} \sigma_{h_{n, i}}} \cdot \exp \left\{-\frac{1}{2 \sigma_{h_{n, i}}^{2}}(h(x)-\theta)\right\}
$$

where $\theta$ is the mean value of noise. Under the condition of minimum mean square error, the variance of the observed noise signal and the prior probability is presented as follows:

$$
\begin{aligned}
\sigma_{h_{n}}^{2} & =\frac{1}{N} \cdot \sum_{k}^{N} h_{n}^{2}(x), \\
\sigma_{\text {prior }}^{2} & =\frac{\widehat{\theta}}{N \sqrt{2 \pi} \sigma_{h_{n}}^{2}} \cdot \sum_{k}^{N} h_{n}(x) \cdot \exp \left\{-\frac{1}{2 \sigma_{h_{n}}^{2}}(\widehat{\theta}-\mu)^{2}\right\} .
\end{aligned}
$$

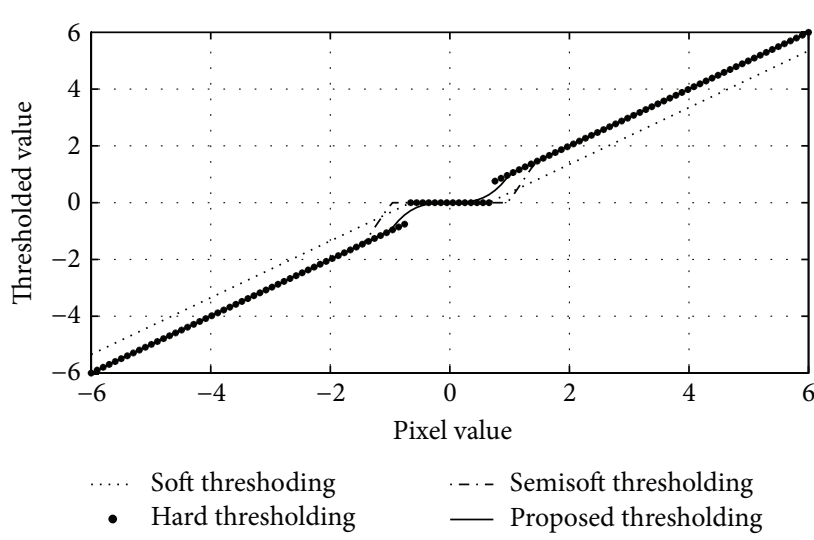

FIgURe 2: Thresholding curves. Dotted line, asterisk line, dash line, and solid line denote soft thresholding, hard thresholding, semisoft thresholding, and the proposed thresholding, respectively.

The Bayesian mean square error (BMSE) of highfrequency wavelet coefficients is as follows:

$$
\mathrm{BMSE}=\iint(\theta-\widehat{\theta})^{2} \cdot P\left(h_{n} \mid \theta\right) d h_{n} d \theta
$$

The Bayesian posterior variance can be calculated when $\mathrm{BMSE}$ reaches to its maximum value, $d \operatorname{BMSE}(\theta) / d \theta=0$ :

$$
\widehat{\sigma}_{h_{n, 0}}^{2}=\sigma_{h_{n}}^{2} \cdot\left(\frac{\sigma_{\text {prior }}^{2}}{\sigma_{h_{n}}^{2}}+\frac{1}{N}\right) .
$$

According to the characteristics of the ultrasonic TOFD data, a new threshold function with the adaptivity to noise is established based on the estimated noise variance to compress the wavelet coefficients. This threshold value for thresholding is as follows:

$$
\lambda= \begin{cases}2 N \times \sqrt{\sigma_{h_{n}}^{2}}, & \max \left(\left|\widehat{\sigma}_{h_{n}}-\sigma_{h_{n}}\right|\right)=0, \\ \frac{\sigma_{h_{n}}^{2},}{\widehat{\sigma}_{h_{n}}} & \max \left(\left|\widehat{\sigma}_{h_{n}}-\sigma_{h_{n}}\right|\right)>0 .\end{cases}
$$

In the condition of the above threshold functions, we give a new self-adaptive thresholding to compress the wavelet coefficients. The wavelet coefficients can be processed as follows and some thresholding curves including the classic thresholding and the proposed one are shown in Figure 2.

$$
h_{n}(x)= \begin{cases}h_{n}^{3}(x), & \left|h_{n}(x)\right| \leq \lambda \\ h_{n}(x), & \left|h_{n}(x)\right|>\lambda\end{cases}
$$

2.3. Image Registration. Ultrasonic TOFD inspection system usually samples one image to determine the size and the depth of the defect quantitatively, and the TOFD images are easy to be contaminated by noise in this procedure. Actually, it is easy to find from multiple TOFD images that the noise is randomly distributed in each image; in addition, each image is obtained with small displacement. Therefore, we adopt image registration and blending them together to eliminate the displacement and the stochastic noise. In order 


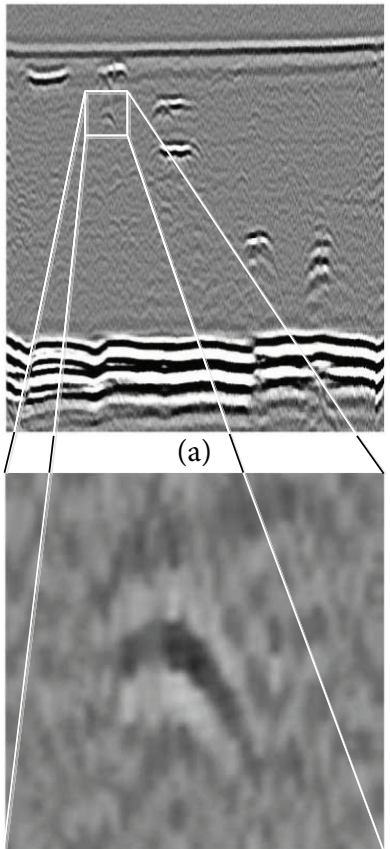

(c)

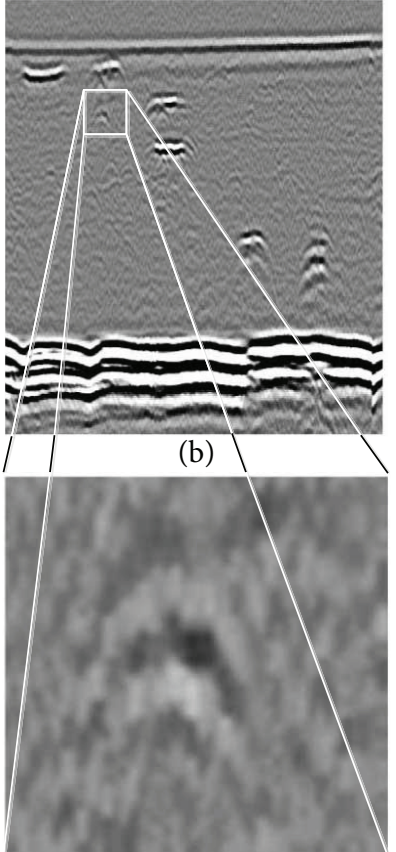

(d)
FIgure 3: The noise distribution in different TOFD images. (a) Original image. (b) The other original image with small displacement of probe during data sampling. (c) and (d) are the zoomed in images of the part framed with white box in (a) and (b), respectively.

to sample the TOFD signal with higher stochasticity, the initial position of probes should be finely tuned each time. Then, the TOFD images with small random displacement can be obtained. Adopting image registration to modify the displacement in each image and the noise can be reduced via blending the registered images together. Thereafter, the quality of TOFD images can be improved.

Figure 3(a) shows one original image sampled by TOFD system. Figure 3(b) shows the other original image with small displacement compared to Figure 3(a). Figures 3(c) and 3(d) are the zoomed in images of the framed part with white box in Figures 3(a) and 3(b), respectively. From Figure 3, the noise in different images has obvious different distributions and varies randomly among images.

In order to eliminate the error lead by the probe displacement in TOFD images, we select one of the TOFD images as the template image or reference image, and the others are the images needed to be registered or the floating image. The error between $f(x, y)$ and $g(x, y)$ can be evaluated by normalized mean square error (NRMSE), presented as follows:

$$
E^{2}=\min _{x_{0}, y_{0}} \frac{\sum_{x, y}\left|g\left(x-x_{0}, y-y_{0}\right)-f(x, y)\right|^{2}}{\sum_{x, y}|f(x, y)|^{2}}
$$

where $\left(x_{0}, y_{0}\right)$ is the position difference in coordinate between $f(x, y)$ and $g(x, y)$. Equation (9) denotes the squared error between $g(x, y)$ after shifting with $\left(x_{0}, y_{0}\right)$

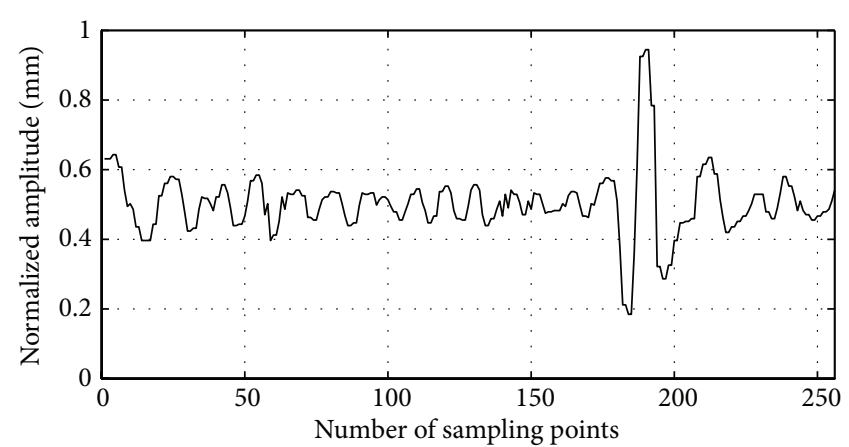

(a)

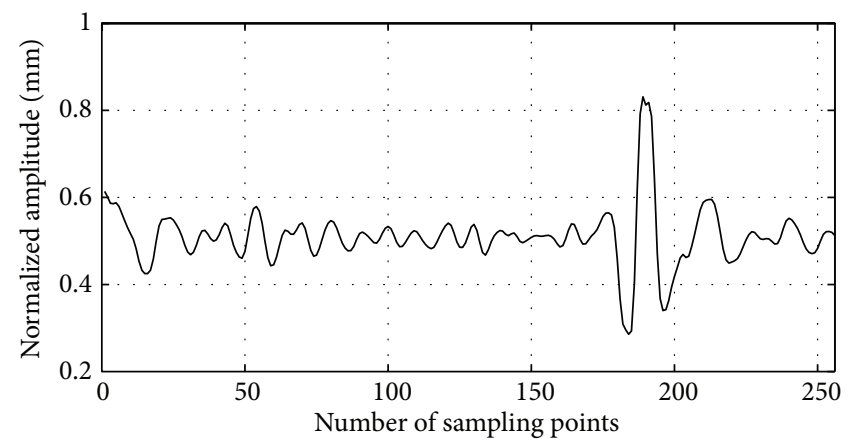

(b)

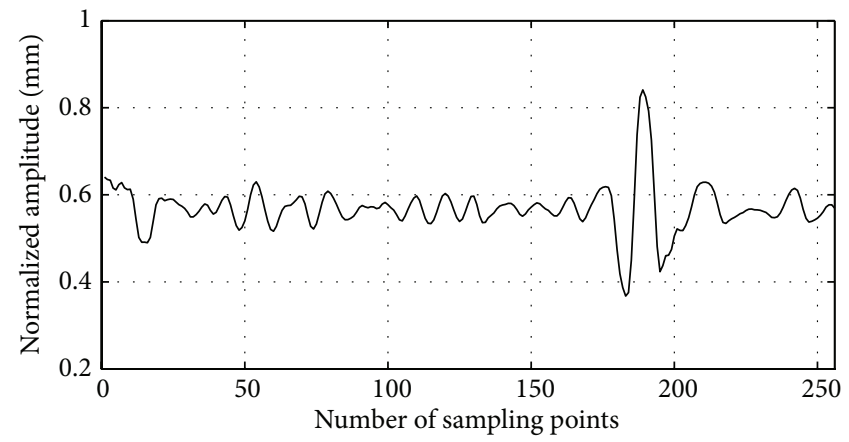

(c)

FIgUre 4: The processed result of A-scan signal by wavelet thresholding. (a) Original signal. (b) The result via soft thresholding. (c) The result via proposed thresholding method.

and $f(x, y)$. After image registration, the square error can be reduced, presented as follows:

$$
E^{2}=1-\frac{\max _{x_{0}, y_{0}}\left|\gamma_{f g}\left(x_{0}, y_{0}\right)\right|^{2}}{\sum_{x, y}|f(x, y)|^{2} \sum_{x, y}|g(x, y)|^{2}},
$$

where $\gamma_{f g}$ is the crosscorrelation. The squared crosscorrelation $\left|\gamma_{f g}(x, y)\right|^{2}$ will reach to the maximum value via image registration. A new image is obtained by using multiple TOFD image registration and blending the registered images $g_{i}\left(x-x_{0}, y-y_{0}\right)$ together, shown in the following equation:

$$
g_{K}(x, y)=\frac{\sum_{i=1}^{K} g_{i}\left(x-x_{0 i}, y-y_{0 i}\right)}{K}
$$



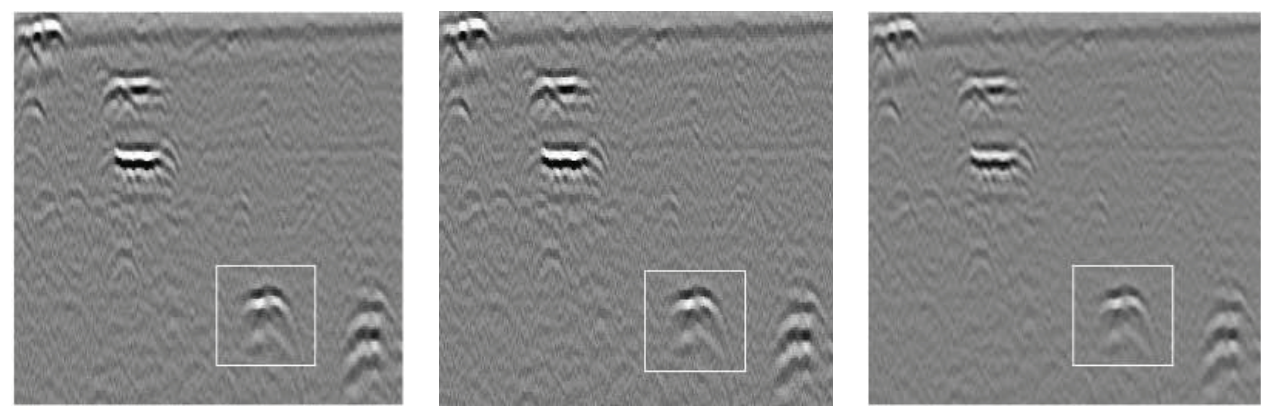

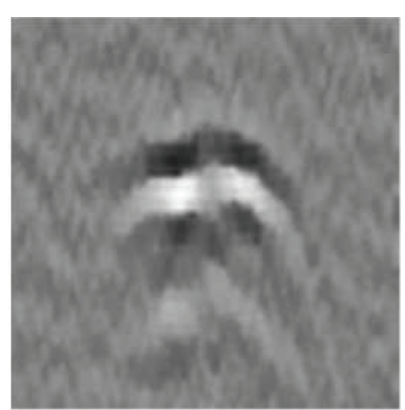

(a)

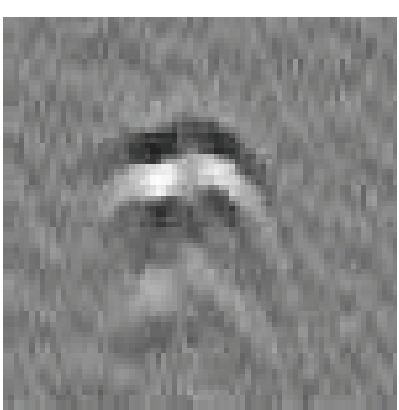

(b)

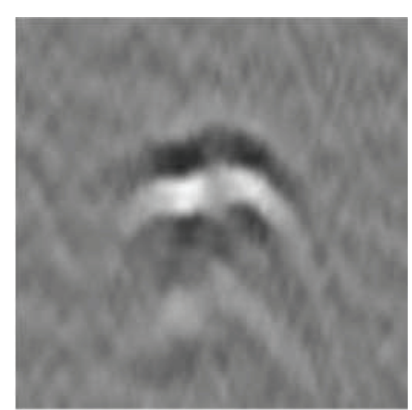

(c)

FIgURE 5: The denoised image via our proposed method. (a) Original TOFD image. (b) Denoised image via soft thresholding. (c) Denoised image via proposed thresholding. The zoomed in image is shown on the right side.

where $K$ denotes the number of images needed to be registered. All the TOFD images are modified as well as being sampled with the same probe position, which means the pixels in each image have the same values but different noise levels and distributions. However, the defect signal keeps retained. The noise level can be weakened by average effect, and the defect signal will be enhanced via blending all the images together.

\section{Experiment Analysis}

The whole experiment system consists of establishing TOFD weld defect inspection system, data sampling, and forming a series of TOFD images. Firstly, we adopt wavelet transform on the raw data for denoising; then, image registration is adopted on the denoised data; lastly, we superimpose all the registered images together to form one new image. The experiment part is realized via TOFD inspection system and data processing. The experiment system is composed of ultrasonic TOFD probes, a scanning device, and specimen with one or more cracks inside. In this paper, we use austenitic steel as the specimen. There are 5 cracks inside the specimen. The size of the specimen is $400 \times 400 \times 30 \mathrm{~mm}$, and we use standard DDENV583-6:2000 probes; the central frequency is $5 \mathrm{MHz}$, the refracted angle is $30^{\circ}$, and the size of the wafer is $6 \mathrm{~mm}$. The gain of the test signal is $63 \mathrm{~dB}-$ $67 \mathrm{~dB}$. The sampling frequency is $100 \mathrm{MHz}$, the refracted angle is $45^{\circ}$, the central moment of the probes is $99 \mathrm{~mm}$, the filter frequency is $5 \mathrm{MHz}$, and water is used as coupling.

In the proceeding of experiment, transmitter and receiver keep a certain distance, and the probe pair moved along the middle line of the weld. The A-scan data is being generated while scanning, adjusting the initial position of the probes before scanning each time; then, the TOFD images with stochastic displacement are obtained. Especially, owing to the randomly setting of the probes' position, the correlation of the noise in each image is rather low.

Before image registration, we adopt two modes of wavelet transform on the original TOFD data: 1D wavelet transform and $2 \mathrm{D}$ wavelet transform. The result of wavelet transform is evaluated by SNR presented in (12). Where SNR is the ratio of signal power and noise power equal to the square deviation ratio of signal and noise.

$$
\mathrm{SNR}=\frac{\sigma_{\text {signal }}^{2}}{\sigma_{\text {noise }}^{2}}=10 \cdot \log _{10}\left(\frac{P_{\text {signal }}}{P_{\text {noise }}}\right) .
$$

Figure 4 shows the result of the A-scan signal processed by wavelet thresholding. Figure 4(a) shows the original signal extracted from the TOFD image. Figure 4(b) shows the result processed by classic thresholding, and the SNR is $19.86 \mathrm{~dB}$. Figure $4(\mathrm{c})$ shows the result processed by the proposed method; the SNR is $26.36 \mathrm{~dB}$. We can see that a better result can be obtained by our proposed method from SNR.

The new image is formed by the composition of multiple denoised A-scan signal, as shown in Figure 5. Figure 5(a) shows the original TOFD image. Figure 5(b) shows the result of the denoised image via soft thresholding, SNR is $12.65 \mathrm{~dB}$, and Figure 5(c) shows the result of the denoised image via the proposed method, SNR is $16.27 \mathrm{~dB}$. The zoomed in image of the framed part by white boxes in Figures $5(\mathrm{a})$ and $5(\mathrm{~b})$ is shown on the right side. From the zoomed in image, the noise distributed around the defect is reduced effectively, so that the image definition is enhanced.

Figure 6 shows the final result of the whole proposed process. Where Figure 6(a) shows the original TOFD image; 

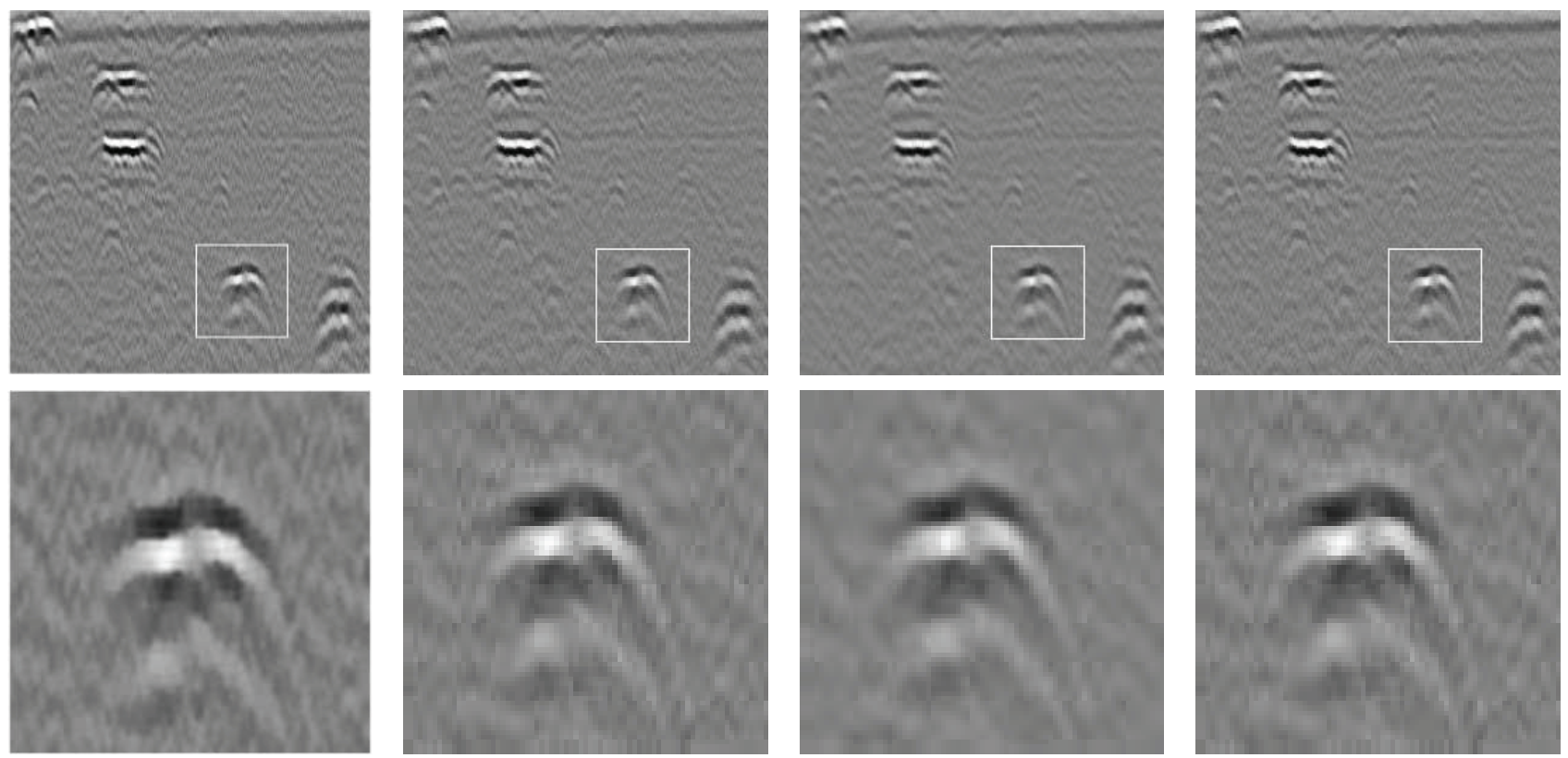

(a)

(b)

(c)

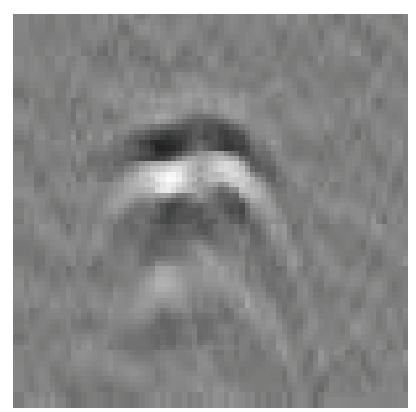

(d)
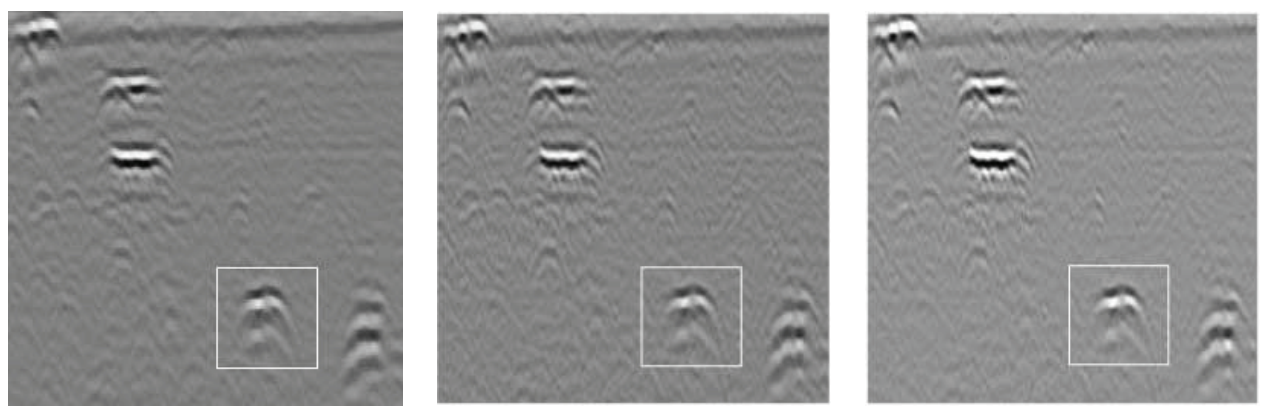

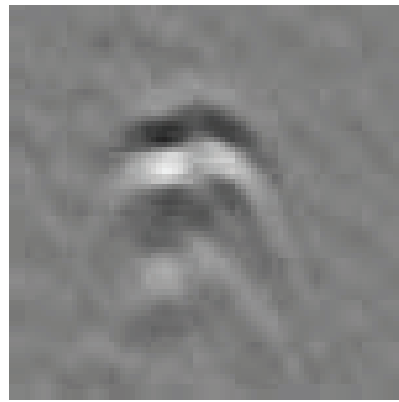

(e)

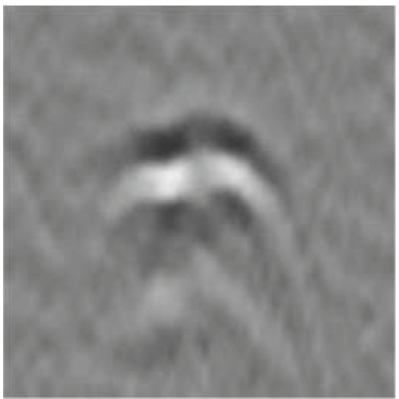

(f)

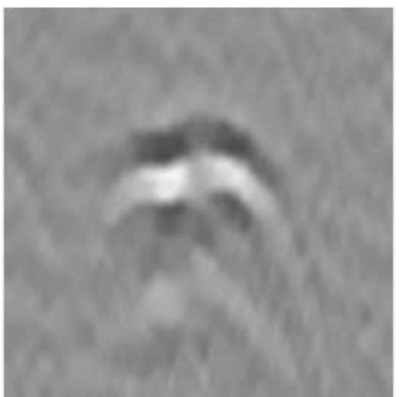

(g)

Figure 6: Multiple TOFD images blending result. (a) Original image. (b) Five superimposed images. (c) 60 superimposed images. (d) Five superimposed registered images obtained via soft thresholding. (e) 60 superimposed registered images obtained via soft thresholding. (f) Five superimposed registered images obtained via our proposed method blending result. (g) 60 superimposed registered images obtained via our proposed method.

Figure 6(b) shows the result of 5 superimposed images and SNR is $12.69 \mathrm{~dB}$; Figure 6(c) shows 60 superimposed images and SNR is $16.81 \mathrm{~dB}$; Figure 6(d) shows 5 superimposed registered images obtained via soft thresholding and SNR is $16.74 \mathrm{~dB}$; Figure $6(\mathrm{e})$ shows the result of 60 superimposed registered images obtained via soft thresholding and SNR is $17.3 \mathrm{~dB}$, while the highest SNR is achieved when the number of superimposed denoised images is 10 , and the corresponding SNR is $18 \mathrm{~dB}$; Figure 6(f) shows the
5 superimposed registered images obtained via our proposed method blending result, and its SNR is $17.46 \mathrm{Db}$; Figure 6(g) shows 60 superimposed registered images obtained via our proposed method and SNR is $23.47 \mathrm{~dB}$.

Figures 7 and 8 shows the curve and the bar chart of the relationship between the SNR and the number of superimposed registered images, respectively. On the one hand, the curve shows that with the increasing of the number of superimposed images, the SNR is increasing as well. The 


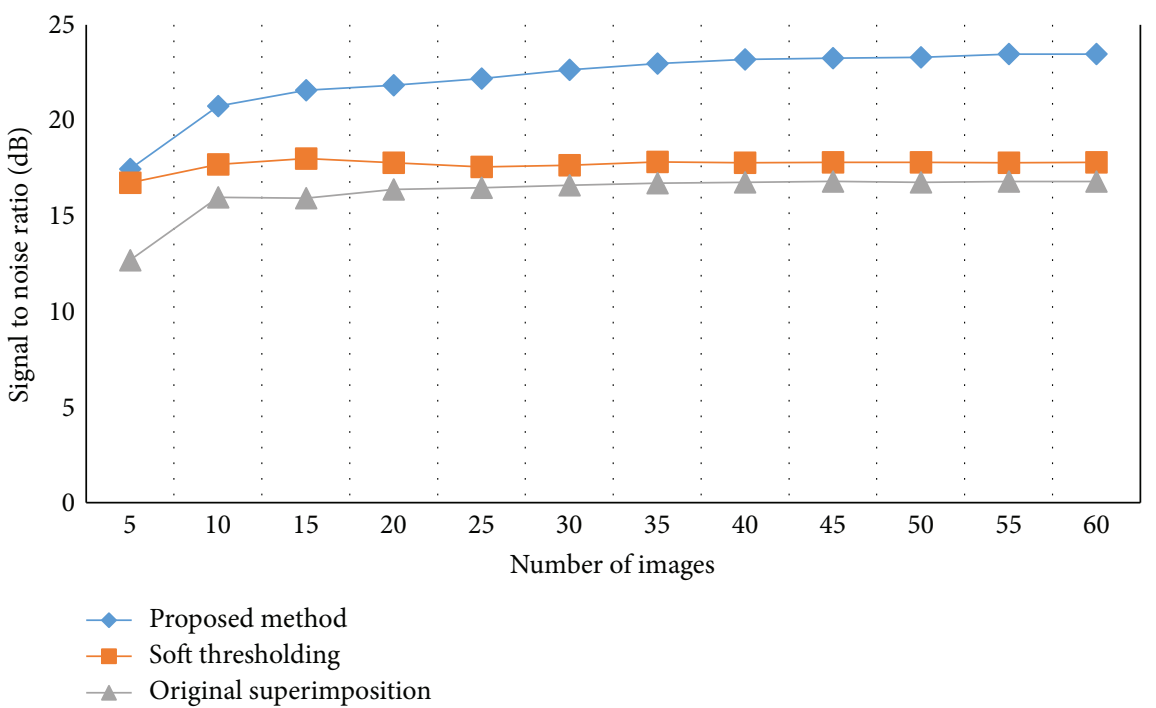

FIGURE 7: The curve of SNR with respect to the number of superimposed images among different methods.

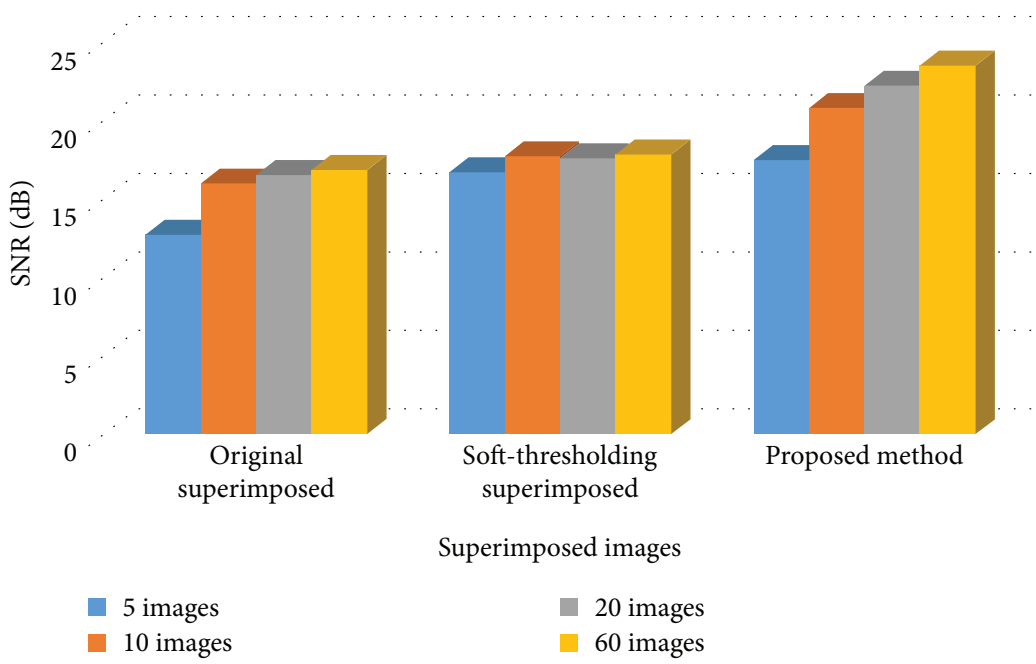

Figure 8: The bar chart of SNR by using different methods.

increasing trend becomes steady when the superimposed number of images is equal or greater than 10. Actually, despite the number of superimposed images is far greater than 10 , SNR only gets a small improvement. Consequently, by only superimposing a relative small number of images, a rather good denoising performance can be obtained, and an image with high SNR is able to form. However, the efficiency of the algorithm is improved. On the other hand, the proposed denoising method achieves the best performance and the SNR is improved about $30 \%$ compared with the others.

\section{Conclusion}

This paper discusses a repeated ultrasonic TOFD inspection method. In so doing, the TOFD A-scan and B-/D-scan signal are characterized with high stochasticity via the random adjustment of the probe pair before each sampling. The average effect impairs the noise to a rather low level via blending all the images together. In order to improve the SNR and the definition of the TOFD images in further step, we adopt 1D wavelet transform and the proposed thresholding method to enhance the SNR of the A-scan signal, which is $39 \mathrm{~dB}$. The denoised TOFD image is composed of multiple denoised A-scan signal. In order to eliminate the displacement among the TOFD images derived from the adjustment of probes, image registration based on maximum correlation is used, thereafter, blending the registered images together. The result shows that SNR got another improvement, from $16.3 \mathrm{~dB}$ to $23.5 \mathrm{~dB}$. The method discussed in this paper is aiming at enhancing the SNR and definition of TOFD images. Indeed, only 10 images are needed to complete the whole image quality enhancement procedure. The method is quite simple and efficient, and it is possible to be applied in timing process in industrial field.

\section{Conflicts of Interest}

The authors declare that they have no conflicts of interest. 


\section{Acknowledgments}

This work was supported by National Nature Science Foundation of China (Grant no. 61471304), and the authors wish to acknowledge them for their support. The authors also thank Southwest Jiaotong University NDT Research Center and Olympus NDT Joint Laboratory of Nondestructive Testing for their kind support in the experiment.

\section{References}

[1] M. G. Silk, "Sizing crack-like defects by ultrasonic means," Research Techniques in Non-Destructive Testing, vol. 3, pp. 51-99, 1977.

[2] S. Baby, T. Balasubramanian, R. J. Pardikar, M. Palaniappan, and R. Subbaratnam, "Time-of-flight diffraction (TOFD) technique for accurate sizing of surface-breaking cracks," Insight Non-Destructive Testing and Condition Monitoring, vol. 45, no. 6, pp. 426-430, 2003.

[3] Y. Zhang, W. Yu, M. J. Zuo, and X. Wang, "Ultrasonic time-of-flight diffraction crack size identification based on cross-correlation," in 2008 Canadian Conference on Electrical and Computer Engineering, pp. 1797-1800, Niagara Falls, ON, Canada, 2008.

[4] S. K. Nath, K. Balasubramaniam, C. V. Krishnamurthy, and B. H. Narayana, "Detection and sizing of defects in complex geometry weld by manual ultrasonic time of flight diffraction inspection," Journal of Pressure Vessel Technology, vol. 131, no. 5, article 051501, 2009.

[5] A. Al-Ataby, W. Al-Nuaimy, C. R. Brett, and O. Zahran, "Automatic detection and classification of weld flaws in TOFD data using wavelet transform and support vector machines," Insight - Non-destructive Testing and Condition Monitoring, vol. 52, no. 11, pp. 597-602, 2010.

[6] H. Zhu, P. Yang, and Y. Cao, "Local optimal threshold technique for the segmentation of ultrasonic time-of-flight diffraction image," Insight - Non-destructive Testing and Condition Monitoring, vol. 53, no. 4, pp. 196-200, 2011.

[7] A. Kechida, R. Drai, and A. Guessoum, "Texture analysis for flaw detection in ultrasonic images," Journal of Nondestructive Evaluation, vol. 31, no. 2, pp. 108-116, 2012.

[8] T. Merazi Meksen, B. Boudraa, R. Drai, and M. Boudraa, "Automatic crack detection and characterization during ultrasonic inspection," Journal of Nondestructive Evaluation, vol. 29, no. 3, pp. 169-174, 2010.

[9] G. Baskaran, C. L. Rao, and K. Balasubramaniam, "Simulation of the TOFD technique using the finite element method," Insight - Non-Destructive Testing and Condition Monitoring, vol. 49, no. 11, pp. 641-646, 2007.

[10] G. Baskaran, K. Balasubramaniam, C. V. Krishnamurthy, and C. Lakshmana Rao, "TOFD IMAGING: ultrasonic TOFD flaw sizing and imaging in thin plates using embedded signal identification technique (ESIT)," Insight - Non-destructive Testing and Condition Monitoring, vol. 46, no. 9, pp. 537-542, 2004.

[11] M. R. Hoseini, M. J. Zuo, and X. Wang, "Denoising ultrasonic pulse-echo signal using two-dimensional analytic wavelet thresholding," Measurement, vol. 45, no. 3, pp. 255-267, 2012.

[12] T. Chen, P. Que, O. Zhang, and Q. Liu, "Ultrasonic nondestructive testing accurate sizing and locating technique based on time-of-flight-diffraction method," Russian Journal of Nondestructive Testing, vol. 41, no. 9, pp. 594-601, 2005.
[13] A. Praveen, K. Vijayarekha, S. T. Abraham, and B. Venkatraman, "Signal quality enhancement using higher order wavelets for ultrasonic TOFD signals from austenitic stainless steel welds," Ultrasonics, vol. 53, no. 7, pp. 1288-1292, 2013.

[14] H. Cygan, P. Aknin, P. Simard, and L. Girardi, "B-scan ultrasonic image analysis for internal rail defect detection," in World Congress on Railway Research (WCRR'03), Edinburgh, UK, 2003.

[15] A. Fathi and A. R. Naghsh-Nilchi, "Efficient image denoising method based on a new adaptive wavelet packet thresholding function," IEEE Transactions on Image Processing, vol. 21, no. 9, pp. 3981-3990, 2012.

[16] J. Huang, T.-Z. Huang, X.-L. Zhao, Z.-B. Xu, and X.-G. Lv, "Two soft-thresholding based iterative algorithms for image deblurring," Information Sciences, vol. 271, pp. 179-195, 2014.

[17] S. G. Mallat, "A theory for multiresolution signal decomposition: the wavelet representation," IEEE Transactions on Pattern Analysis and Machine Intelligence, vol. 11, no. 7, pp. 674-693, 1989. 


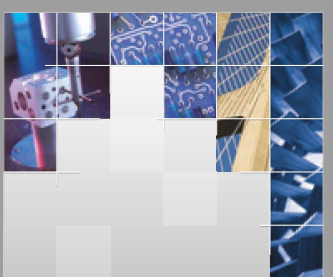

\section{Enfincering}
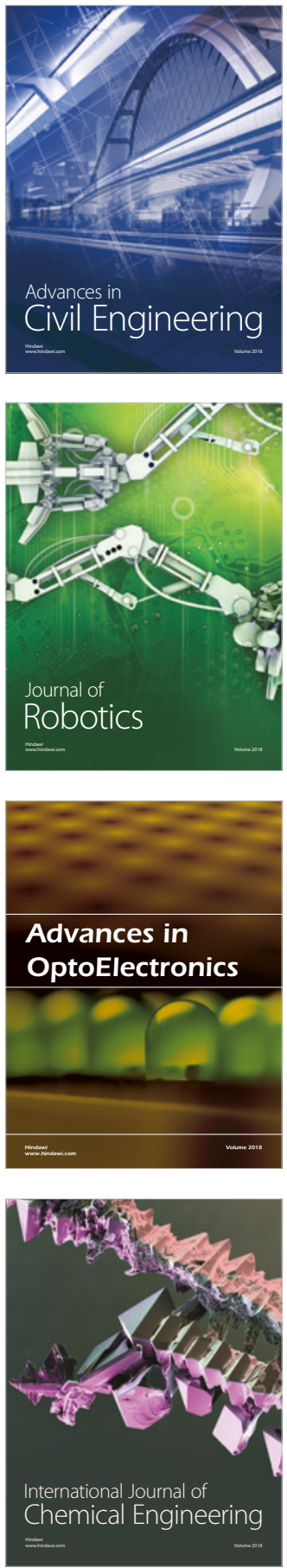

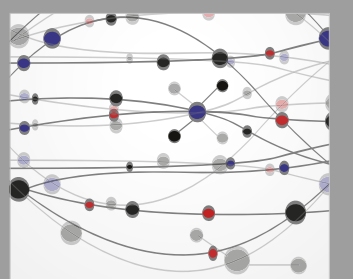

\section{Rotating \\ Machinery}

The Scientific World Journal

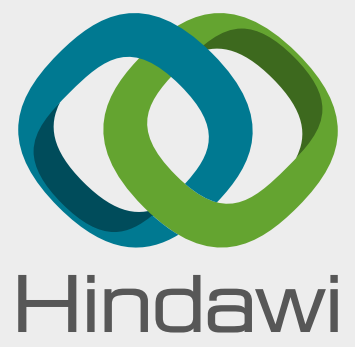

Submit your manuscripts at

www.hindawi.com
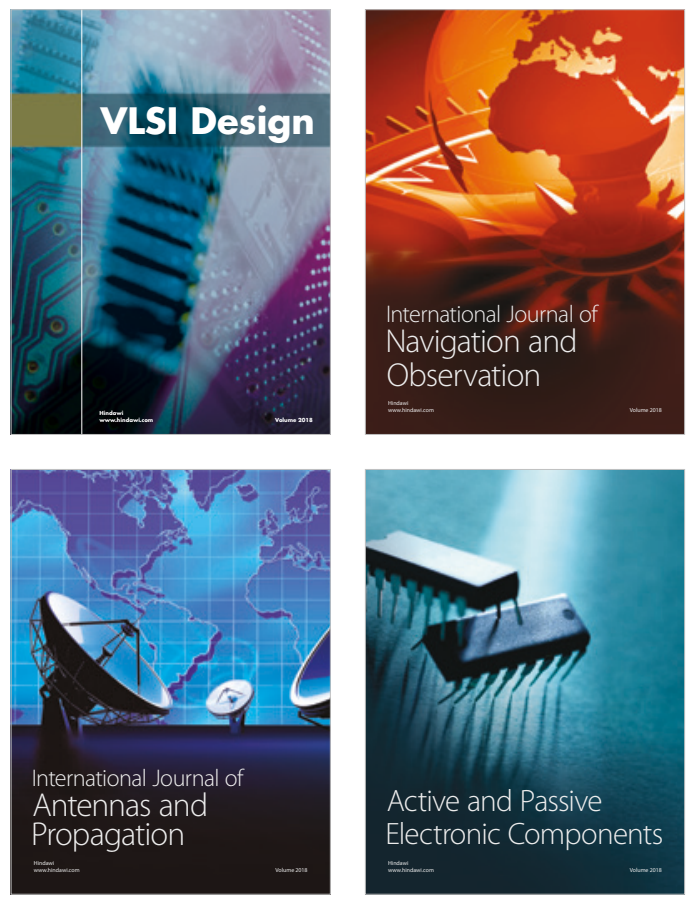
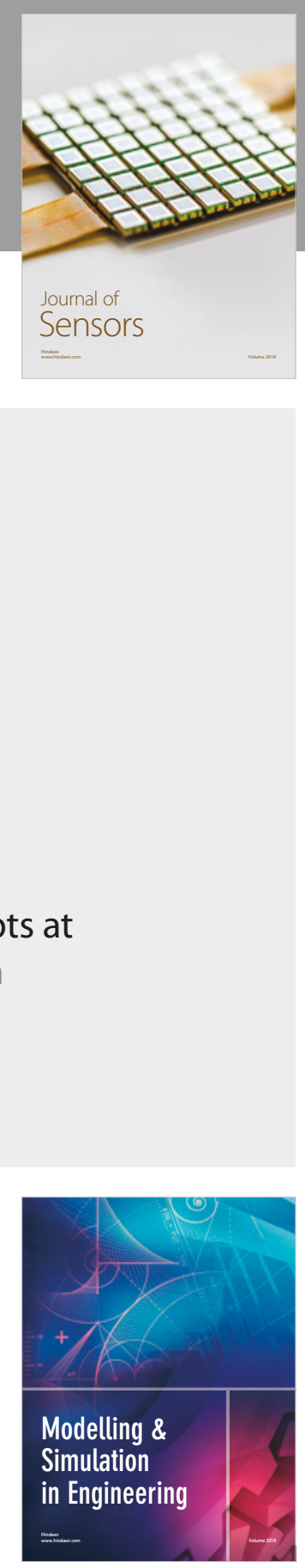

\section{Advances \\ Multimedia}
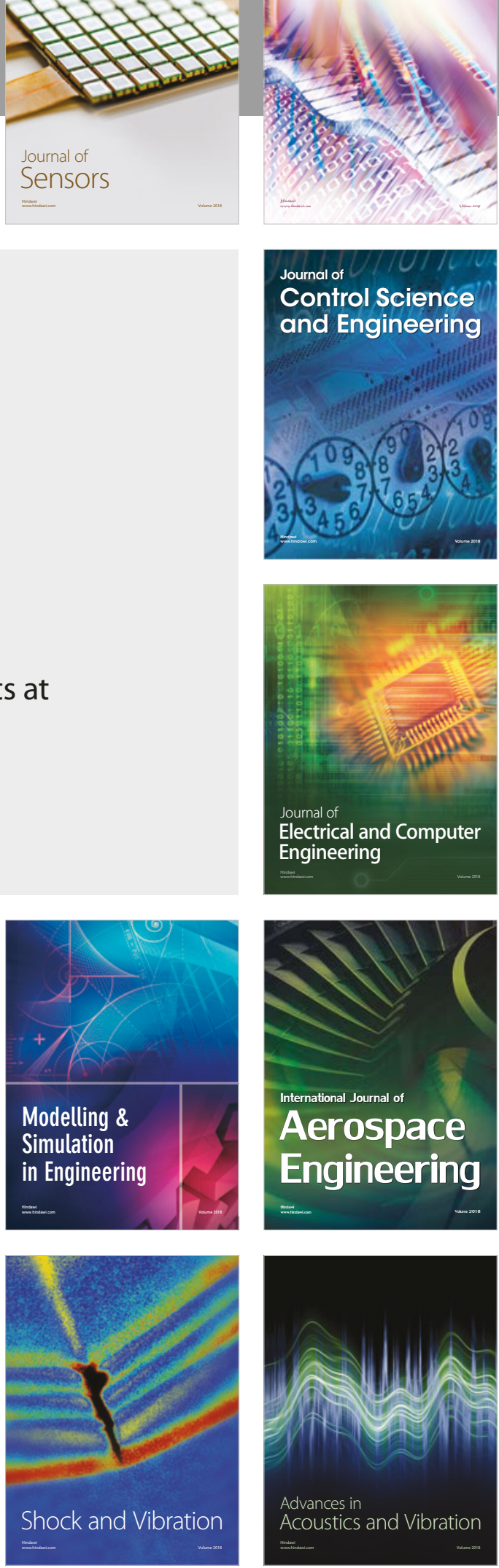